\title{
Quantum Critical Point of Itinerant Antiferromagnet in Heavy Fermion
}

\author{
Hiroaki Kadowaki, ${ }^{1}$ Yoshikazu Tabata, ${ }^{2}$ Masugu Sato, ${ }^{3}$ Naofumi Aso, ${ }^{4}$ Stephane Raymond, ${ }^{5}$ and Shuzo Kawarazaki ${ }^{2}$ \\ ${ }^{1}$ Department of Physics, Tokyo Metropolitan University, Hachioji-shi, Tokyo 192-0397, Japan \\ ${ }^{2}$ Graduate School of Science, Osaka University, Toyonaka, Osaka 560-0043, Japan \\ ${ }^{3}$ MSD, JASRI, 1-1-1 Kouto Mikazuki-cho Sayo-gun, Hyogo 679-5198, Japan \\ ${ }^{4}$ NSL, Institute for Solid State Physics, University of Tokyo, Tokai, Ibaraki 319-1106, Japan \\ ${ }^{5}$ CEA-Grenoble, DSM/DRFMC/SPSMS, 38054 Grenoble, France
}

(Dated: November 11, 2018)

\begin{abstract}
A quantum critical point of the heavy fermion $\mathrm{Ce}\left(\mathrm{Ru}_{1-x} \mathrm{Rh}_{x}\right)_{2} \mathrm{Si}_{2}(x=0,0.03)$ has been studied by single-crystalline neutron scattering. By accurately measuring the dynamical susceptibility at the antiferromagnetic wave vector $\boldsymbol{k}_{3}=0.35 \boldsymbol{c}^{*}$, we have shown that the energy width $\Gamma\left(\boldsymbol{k}_{3}\right)$, i.e., inverse correlation time, depends on temperature as $\Gamma\left(\boldsymbol{k}_{3}\right)=c_{1}+c_{2} T^{3 / 2 \pm 0.1}$, where $c_{1}$ and $c_{2}$ are $x$ dependent constants, in a low temperature range. This critical exponent $3 / 2 \pm 0.1$ proves that the quantum critical point is controlled by that of the itinerant antiferromagnet.
\end{abstract}

PACS numbers: 71.27.+a, 71.10.Hf, 75.30.Mb, 75.40.Gb

Quantum critical points (QCP) separating ferromagnetic or antiferromagnetic states from paramagnetic Fermi liquid states in strongly correlated electron systems have been investigated for decades. Successful descriptions of critical behavior close to QCPs were traditionally provided by the self-consistent renormalization (SCR) theory of spin fluctuations [1, 2] for $d$-electron systems based on the Hubbard model. The mean-field-type approximations used in the SCR theory were justified by the renormalization group studies [3] based on the Hertz effective action above upper critical dimensions. For the ferromagnetic QCP, theoretical predictions were supported by experimental studies of $d$-electron systems [1, 4]. In contrast there is little experimental understanding of the antiferromagnetic QCP [2].

A recent intriguing issue of $\mathrm{QCP}$ under controversial debate is directed toward revealing relevant fixed points for antiferromagnetic QCPs in heavy-fermion systems [5]. In energy scales much lower than Kondo temperature $T_{\mathrm{K}}$, $f$ and conduction electrons form composite quasiparticles with a large mass renormalization in paramagnetic heavy fermions. By tuning a certain parameter, e.g., pressure or concentration, an antiferromagnetic long range order emerges from the Fermi liquid state. In a weak coupling picture, it has been hypothesized that the same QCP as the $d$-electron itinerant antiferromagnet, referred to as spin density wave (SDW) type $\mathrm{QCP}$, is relevant to the heavy fermion QCP [5, $[$ ] $]$.

However despite a number of experimental studies of heavy-fermion systems showing non-Fermi liquid behavior, none of them definitely supports the SDW QCP [7, 8, 9]. This stems partly from experimental difficulty in measuring weakly divergent quantities around QCP especially for bulk properties, which has been also the case for $d$-electron itinerant antiferromagnets [2]. On the contrary, several recent experiments suggest the possibility of a novel strong coupling picture of the QCP [5, 10, 11]. Among these studies direct measurements of the diverg- ing spin fluctuation using single-crystalline neutron scattering for the heavy fermion $\mathrm{CeCu}_{5.9} \mathrm{Au}_{0.1}$ provided interesting insight [10]. On the basis of the observed $E / T$ scaling with an anomalous exponent [10] and effective two space dimensions 12], a scenario of a locally critical QCP was proposed [5, 13. In contrast to the SDW $\mathrm{QCP}$, this theory stresses that separation of $f$ spin from the quasiparticle state occurs abruptly at the QCP.

In the present work, we have studied an antiferromagnetic QCP of another heavy-fermion system $\mathrm{Ce}\left(\mathrm{Ru}_{1-x} \mathrm{Rh}_{x}\right)_{2} \mathrm{Si}_{2}(x=0,0.03)$ using single-crystalline neutron scattering. Stoichiometric $\mathrm{CeRu}_{2} \mathrm{Si}_{2}$ is an archetypal paramagnetic heavy-fermion with enhanced $C / T \simeq 350 \mathrm{~mJ} / \mathrm{K}^{2} \mathrm{~mol}$ and $T_{\mathrm{K}} \simeq 24 \mathrm{~K}$ 14]. Extensive neutron scattering studies of $\mathrm{CeRu}_{2} \mathrm{Si}_{2}$ [15] have shown that spin fluctuations possessing three-dimensional $(d=$ 3) character are excellently described by the SCR theory for heavy fermions [ 6 . A small amount of $\mathrm{Rh}$ doping $x>x_{\mathrm{c}} \simeq 0.04[16]$ induces an antiferromagnetic phase [see Fig. [1(a)] of the sinusoidally modulated structure with the wave vector $\boldsymbol{k}_{3}=0.35 \boldsymbol{c}^{*}[17$.

The Rh doping modifies exchange interactions, while keeping $T_{\mathrm{K}}$ constant 16], and brings about the antiferromagnetic phase in the concentration range $x_{\mathrm{c}}<x<0.35$. The dome structure of this phase and another antiferromagnetic phase with the modulation vector $\left(\frac{1}{2} \frac{1}{2} 0\right)$ in the higher concentration range $0.6<x$ suggests a certain frustration effect among exchange interactions. Samples nearly tuned to the lowest concentration QCP $\left(x \sim x_{\mathrm{c}}\right)$ show non-divergent $C / T(T \rightarrow 0)[18]$ and $\Delta \rho \propto T^{3 / 2}$ [see Fig. 1(b)], which are consistent with the SDW QCP in $d=3$. Thus one can expect that $\mathrm{Ce}\left(\mathrm{Ru}_{1-x} \mathrm{Rh}_{x}\right)_{2} \mathrm{Si}_{2}$ $\left(x \lesssim x_{\mathrm{c}}\right)$ is suited to investigate the SDW QCP without disorder effects. On the other hand, non-Fermi liquid behavior observed in the higher concentration range $0.35<x$ was reported to be influenced by disorders [19, 20].

In order to experimentally show the SDW QCP in 
$d=3$, spin correlation studied by the renormalization group theory [3, 21] should be measured directly by neutron scattering. The theory of the SDW QCP shows that the wave-vector dependent susceptibility for the tuned sample $\left(x=x_{\mathrm{c}}\right)$ diverges as $\chi\left(\boldsymbol{k}_{3}\right) \propto T^{-3 / 2}[1,[3]$, or the characteristic energy of the spin fluctuation, i.e., the inverse correlation time, depends on temperature as $\Gamma\left(\boldsymbol{k}_{3}\right) \propto \chi\left(\boldsymbol{k}_{3}\right)^{-1} \propto T^{3 / 2}$. By taking the detuning effect $\left(x<x_{\mathrm{c}}\right)$ into account, leading two terms of $\Gamma\left(\boldsymbol{k}_{3}\right)$ computed by the renormalization group theory [3, 21] are given by

$$
\Gamma\left(\boldsymbol{k}_{3}\right)=c_{1}+c_{2} T^{3 / 2},
$$

where $c_{1}$ and $c_{2}$ are $x$ dependent constants. This equation is an approximation in the temperature range $T_{\mathrm{FL}} \ll$ $T \ll T_{\mathrm{K}}$, where $T_{\mathrm{FL}}$ is a crossover temperature below which system shows the Fermi liquid behavior [3, 21]. In the present work, we have accurately measured $\Gamma\left(\boldsymbol{k}_{3}\right)$ and have shown that it agrees well with Eq. (11) for both the nearly tuned sample $x=0.03$ and the stoichiometric sample $x=0$, which indicates that disorder does not influence the critical behavior.

Neutron-scattering measurements were performed on the triple-axis spectrometer HER at the Japan Atomic Energy Research Institute. It was operated using final energies of $E_{\mathrm{f}}=3.1$ and $2.4 \mathrm{meV}$ providing energy resolutions of 0.1 and $0.05 \mathrm{meV}$ (full width at half maximum), respectively, at elastic positions. Single crystals with a total weight of $19 \mathrm{~g}(x=0)$ and $17 \mathrm{~g}(x=0.03)$ were grown by the Czochralski method. Two sets of multicrystal samples aligned together were mounted in a $\mathrm{He}$ flow cryostat so as to measure a $(h 0 l)$ scattering plane. All the data shown are converted to the dynamical susceptibility. It is scaled to absolute units by comparison with the intensity of a standard vanadium sample. We note that a new point of the present work is unprecedented experimental accuracy in determining the critical exponent $[3 / 2$ in Eq. (11)] using large samples and long counting time. This has enabled us to determine the singularity of $\mathrm{QCP}$ and to make qualitative conclusion of the universality class. In the pioneering work using the related compound $\mathrm{Ce}_{1-x} \mathrm{La}_{x} \mathrm{Ru}_{2} \mathrm{Si}_{2}$ [8, 22], Eq. (11) has been discussed assuming the fixed value of the exponent, which could be determined only with an order larger experimental error.

The imaginary part of the dynamical susceptibility at $\boldsymbol{Q}=\boldsymbol{k}_{3}+\boldsymbol{q}$ with small $|\boldsymbol{q}|$ and $|E|$ is predicted to be approximated by

$$
\begin{aligned}
\operatorname{Im} \chi\left(\boldsymbol{k}_{3}+\boldsymbol{q}, E\right) & =\frac{\chi\left(\boldsymbol{k}_{3}\right) \Gamma\left(\boldsymbol{k}_{3}\right) E}{E^{2}+\Gamma\left(\boldsymbol{k}_{3}+\boldsymbol{q}\right)^{2}}, \\
\Gamma\left(\boldsymbol{k}_{3}+\boldsymbol{q}\right) & =D_{c}\left[\kappa_{c}^{2}+q_{c}^{2}+F\left(q_{a}^{2}+q_{b}^{2}\right)\right],
\end{aligned}
$$

where $D_{c}$ and $F$ are $T$ independent parameters, and $\kappa_{c}$ is inverse correlation length along the $c$ axis [23]. This expansion form with the $T$ independent product

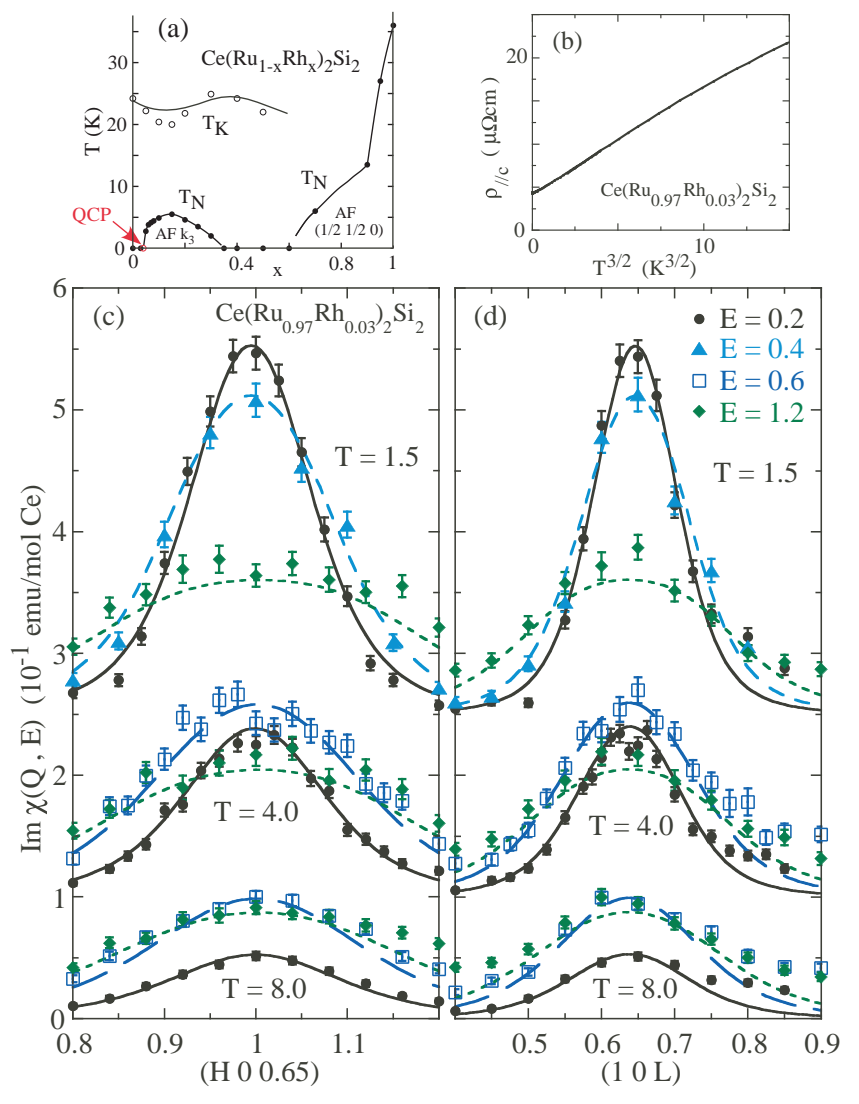

FIG. 1: (color online) (a) The phase diagram and $T_{\mathrm{K}}$ of $\mathrm{Ce}\left(\mathrm{Ru}_{1-x} \mathrm{Rh}_{x}\right)_{2} \mathrm{Si}_{2}$ are reproduced from Refs. [16, 17]. (b) Resistivity of the sample with $x=0.03$ is plotted as a function of $T^{3 / 2}$. Constant- $E$ scans taken with $E=0.2,0.4,0.6$ and $1.2 \mathrm{meV}$ along (c) $\boldsymbol{Q}=(H, 0,0.65)$ and (d) $(1,0, L)$ lines for the sample with $x=0.03$. Data of $T=1.5$ and $4 \mathrm{~K}$ are shifted by 0.25 and $0.1 \mathrm{emu} / \mathrm{mol} \mathrm{Ce}$, respectively for clarity. Curves in (c) and (d) are calculations using Eqs. (2a) and (2b), corrected for resolution functions with the same fit parameter as those shown in Fig. 2

$\chi\left(\boldsymbol{k}_{3}\right) \Gamma\left(\boldsymbol{k}_{3}\right)$ has been used in the SCR [2, 6] and renormalization group [21] theories. The two parameters $D_{c}$ and $F$ were determined by using constant- $Q$ and $-E$ scans for both samples with $x=0$ [15] and 0.03 at $T=1.5$ K. These data were fitted to Eqs. (2a) and (2b) convolved with the resolution functions. In Figs. 1(c) and 1(d) we show constant- $E$ scans through the antiferromagnetic wave vector $\boldsymbol{Q}=(101)-\boldsymbol{k}_{3}$ and the fit curves for the sample with $x=0.03$. The good quality of fitting indicates that Eqs. (2a) and (2b) well describe the experimental data at $T=1.5 \mathrm{~K}$. We obtained concentration independent values of the parameters $D_{c}=98 \pm 4(\mathrm{meV}$ $\AA^{2}$ ) and $F=0.12 \pm 0.01$.

Temperature dependence of $\Gamma\left(\boldsymbol{k}_{3}\right)=D_{c} \kappa_{c}^{2}$ has been determined by performing constant- $Q$ scans taken at $\boldsymbol{Q}=(101)-\boldsymbol{k}_{3}$ with much higher statistical accuracy than previous measurements [15, 22]. These scan data 
were fitted to Eq. (2a) convolved with the resolution functions, where there are two adjustable parameters $\Gamma\left(\boldsymbol{k}_{3}\right)$ and $\chi\left(\boldsymbol{k}_{3}\right)$. Several fit results of the constant- $Q$ scans for the samples with $x=0$ and 0.03 are shown in Fig. 2 From these figures one can see that the quality of fitting is excellent. We also checked the $T$ independence of the parameters $D_{c}$ and $F$ by comparing the constant- $E$ scans in Fig. 1 at $T=4$ and $8 \mathrm{~K}$ with those calculated using the $T$ dependent $\Gamma\left(\boldsymbol{k}_{3}\right)$ and $\chi\left(\boldsymbol{k}_{3}\right)$ determined by the constant- $Q$ scans. The calculated curves in Fig. [1 which have no adjustable parameters for $T=4$ and 8 $\mathrm{K}$, agree reasonably well with the observations. Thus we conclude that the theoretical approximation of Eqs. (2) has been experimentally confirmed, and that the fit parameter $\Gamma\left(\boldsymbol{k}_{3}\right)$ has been determined very precisely.

Temperature dependence of $\Gamma\left(\boldsymbol{k}_{3}\right)$ is shown in Fig. 3 by plotting data as a function of $T^{3 / 2}$. At low temperatures observed data clearly agree with the linear behavior of Eq. (11). In fact, by least squares fitting we obtained $\Gamma\left(\boldsymbol{k}_{3}\right)=(0.67 \pm 0.01)+(0.0095 \pm 0.0021) T^{1.53 \pm 0.08}$ (in units of meV) in $1.5<T<16 \mathrm{~K}$ for the sample with $x=$ 0 and $\Gamma\left(\boldsymbol{k}_{3}\right)=(0.129 \pm 0.007)+(0.020 \pm 0.003) T^{1.49 \pm 0.07}$ in $1.5<T<8 \mathrm{~K}$ for $x=0.03$. Therefore we conclude that the observed critical exponent $3 / 2 \pm 0.1$ is in agreement with the theoretical value $3 / 2$, and consequently that the temperature dependence of the spin fluctuation is controlled by the SDW QCP in $d=3$. The same exponent for both $x=0$ and 0.03 samples ensures that the randomness due to $\mathrm{Rh}$ doping does not affect the criticality. The temperature independence of $\chi\left(\boldsymbol{k}_{3}\right) \Gamma\left(\boldsymbol{k}_{3}\right)$ was also confirmed as shown in the inset of Fig. [3.

The constant $c_{1}$ in Eq. (1) is proportional to the theoretical tuning parameter, a coefficient of the quadratic terms of the Hertz effective action, and $c_{1} \propto x_{\mathrm{c}}-x$ is normally assumed [3]. This assumption is consistent with the observed values of $c_{1}$ and the critical concentration $x_{\mathrm{c}}=0.04 \pm 0.005$. In contrast to this agreement, the concentration dependence of $c_{2}, c_{2}(x=0.03) / c_{2}(x=0) \sim 2$, may suggest a certain difficulty in the theoretical interpretation. The constant $c_{2}$, being proportional to the coefficient of the quartic term of the Hertz effective action, is usually supposed to vary slowly in the concentration range of interest [3]. The appreciable variation of $c_{2}$ seems to suggest unknown perturbations for $\mathrm{Ce}\left(\mathrm{Ru}_{1-x} \mathrm{Rh}_{x}\right)_{2} \mathrm{Si}_{2}$. In terms of the SCR theories, the variation of $c_{2}$ may be accounted for by the adjustable mode-mode coupling constant $\left(\propto c_{2}\right)$ that is used in the phenomenological SCR theory developed for $d$-electron systems [1, 2]. Despite this problem, we think that the critical exponent $3 / 2$ determined by basic characteristics of the system, the space dimension $d=3$ and the dynamical exponent $z=2$, is more important and decisive to conclude the nature of the QCP.

An advantage of the present neutron scattering study is that Eq. (11) holds in a wider temperature range compared to those of indirect measurements using bulk properties,
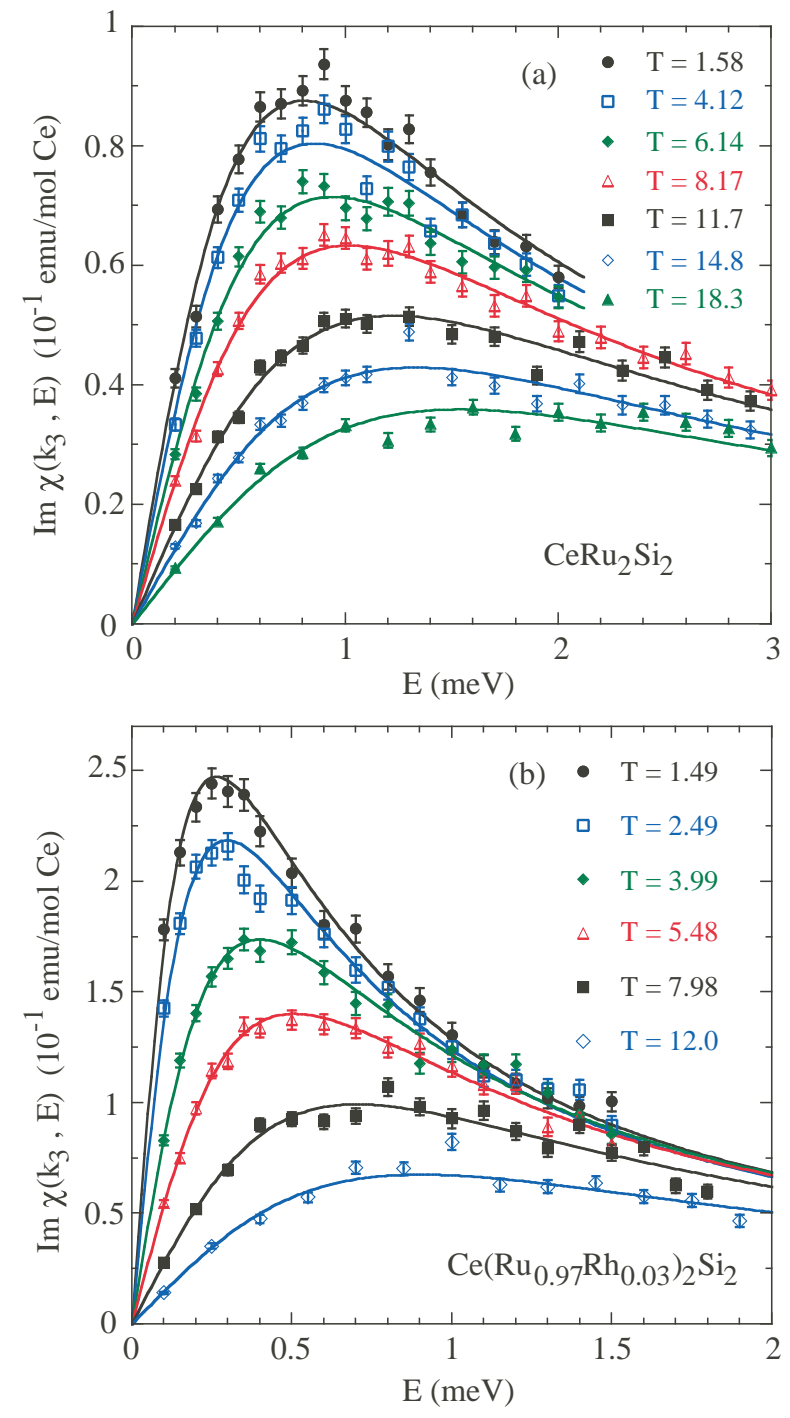

FIG. 2: (color online) Constant- $Q$ scans measured at the antiferromagnetic wave vector $\boldsymbol{Q}=(101)-\boldsymbol{k}_{3}$ for the samples with $x=0$ (a) and $x=0.03$ (b). Curves are fit results using Eq. (2a) with two adjustable parameters $\Gamma\left(\boldsymbol{k}_{3}\right)$ and $\chi\left(\boldsymbol{k}_{3}\right)$.

e.g., $C / T=\gamma_{0}-\alpha T^{1 / 2}$ or $\Delta \rho \propto T^{3 / 2}[8$, 18]. Theoretically Eq. (1) is an approximation in the temperature range $T_{\mathrm{FL}} \ll T \ll T_{\mathrm{K}}$, where $T_{\mathrm{FL}}$ is the crossover temperature to the Fermi liquid state [3, 21]. The temperature range in which Eq. (11) is observable can be discussed quantitatively using the SCR theory [6]. In Fig. 3 the dashed line reproduces the SCR computation of $\Gamma\left(\boldsymbol{k}_{3}\right)$ for $\mathrm{CeRu}_{2} \mathrm{Si}_{2}$ based on the previous neutron scattering study [15]. Apart from discrepancy of the coefficient $c_{2}$, one can see that the $T^{3 / 2}$ dependence of Eq. (1) is a good approximation for the SCR curve in the $T$ range $2.5<T<13 \mathrm{~K}\left(4<T^{3 / 2}<47\right)$, which agrees with that of the observed data for $\mathrm{CeRu}_{2} \mathrm{Si}_{2}$. Since the lower bound temperature is shown to be proportional to the tuning parameter by the renormalization group theory [3, 21], 


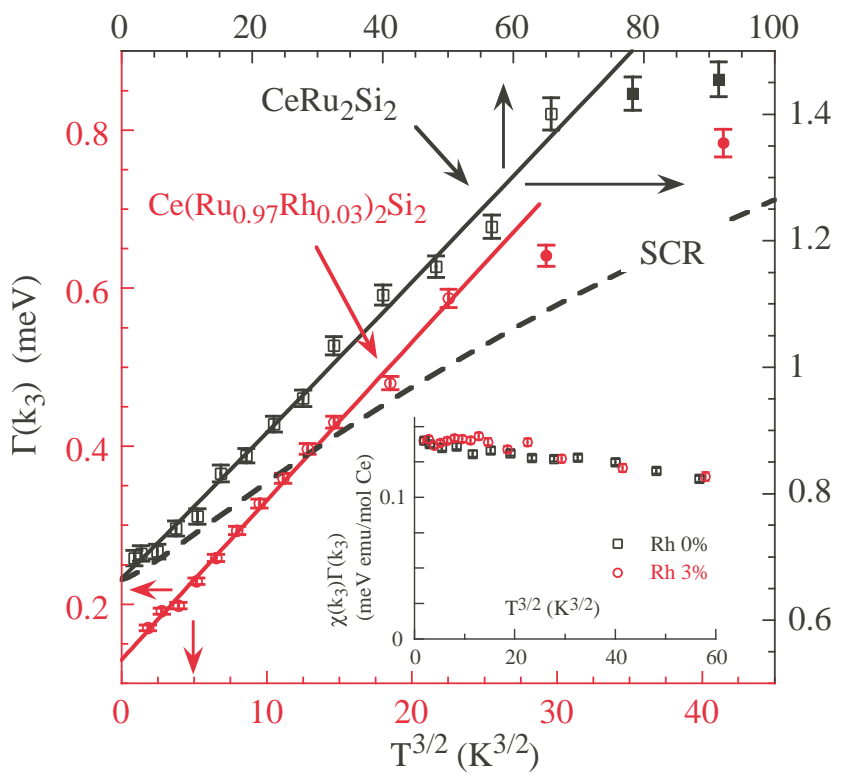

FIG. 3: (color online) Energy width $\Gamma\left(\boldsymbol{k}_{3}\right)$ of the Lorentzian form Eq. (2a) is plotted as a function of $T^{3 / 2}$. Full lines are fit to $\Gamma\left(\boldsymbol{k}_{3}\right)=c_{1}+c_{2} T^{v}$ with adjustable parameters $c_{1}, c_{2}$, and $v$ in low temperature ranges, where data are displayed by open symbols. The dashed line is the calculation [15] using the SCR theory [6]. The inset shows temperature dependence of the product $\chi\left(\boldsymbol{k}_{3}\right) \Gamma\left(\boldsymbol{k}_{3}\right)$.

the $T^{3 / 2}$ dependence can be expected in a $T$ range of 0.5 $\mathrm{K}<T\left(0.35<T^{3 / 2}\right)$ for the sample with $x=0.03$. The smaller $T$ range of the $T^{3 / 2}$ dependence for $x=0.03$ is probably related to the larger constant $c_{2}(x=0.03)$. We note that below $2.5 \mathrm{~K}$ the SCR computation of $\Gamma\left(\boldsymbol{k}_{3}\right)$ for $x=0[15]$ is approximated by the Fermi liquid behavior of $\Gamma\left(\boldsymbol{k}_{3}, T\right)-\Gamma\left(\boldsymbol{k}_{3}, 0\right) \propto T^{2}$ [21], which is not clearly seen within the present experimental error.

In connection with neutron scattering experiments of $\mathrm{CeCu}_{5.9} \mathrm{Au}_{0.1}$ 10, 12], it was theoretically predicted [13] that the locally critical QCP is relevant for the twodimensional spin fluctuation, in agreement with the experiments of $\mathrm{CeCu}_{5.9} \mathrm{Au}_{0.1}$. This theory also predicted that the SDW QCP is relevant for the three-dimensional spin fluctuation, which is in accord with the present results. Finally we note that the present work is first clear experimental verification of the SDW QCP to our knowledge among single-crystalline neutron scattering studies on QCP or non-Fermi liquid behavior of heavy fermions, e.g., Refs. 10, 12, 22, 24, 25, 26] and $d$-electron systems, e.g., Refs. 27, 28]. Assuming that criticalities of QCPs are classified into a limited number of universality classes, we expect that the SDW QCP remains to be observed in other systems.

In summary, we have demonstrated that the quantum critical behavior of the heavy fermion $\mathrm{Ce}\left(\mathrm{Ru}_{1-x} \mathrm{Rh}_{x}\right)_{2} \mathrm{Si}_{2}$ is controlled by the SDW type QCP in three space dimen- sions. The inverse correlation time, i.e., energy width $\Gamma\left(\boldsymbol{k}_{3}\right)$ of the dynamical susceptibility, shows the $T^{3 / 2}$ dependence predicted by the renormalization group and SCR theories.

We wish to acknowledge B. Fåk, J. Flouquet, T. Taniguchi, and T. Moriya for valuable discussions.

[1] T. Moriya, Spin Fluctuations in Itinerant Electron Magnetism (Springer-Verlag, Berlin, 1985).

[2] T. Moriya and K. Ueda, Rep. Prog. Phys. 66, 1299 (2003); Adv. Phys. 49, 555, (2000).

[3] J. A. Hertz, Phys. Rev. B 14, 1165 (1976); A. J. Millis, ibid. 48, 7183 (1993).

[4] S. R. Julian, C. Pfleiderer, F. M. Grosche, N. D. Mathur, G. J. McMullan, A. J. Diver, I. R. Walker, and G. G. Lonzarich, J. Phys. Condens. Matter 8, 9675 (1996).

[5] P. Coleman, C. Pépin, Q. Si, and R. Ramazashvili, J. Phys. Condens. Matter 13, R723 (2001).

[6] T. Moriya and T. Takimoto, J. Phys. Soc. Jpn. 64, 960 (1995).

[7] H. v. Löhneysen, T. Pietrus, G. Portisch, H. G. Schlager, A. Schröder, M. Sieck, and T. Trappmann, Phys. Rev. Lett. 72, 3262 (1994).

[8] S. Kambe, S. Raymond, L. P. Regnault, J. Flouquet, P. Lejay, and P. Haen, J. Phys. Soc. Jpn. 65, 3294 (1996).

[9] G. R. Stewart, Rev. Mod. Phys. 73, 797 (2001).

[10] A. Schröder, G. Aeppli, R. Coldea, M. Adams, O. Stockert, H. v. Löhneysen, E. Bucher, R. Ramazashvili, and P. Coleman, Nature (London) 407, 351 (2000).

[11] P. Paschen, T. Lühman, S. Wirth, P. Gegenwart, O. Trovarelli, C. Geibel, F. Steglich, P. Coleman, and Q. Si, Nature (London) 432, 881 (2004).

[12] O. Stockert, H. v. Löhneysen, A. Rosch, N. Pyka, and M. Loewenhaupt, Phys. Rev. Lett. 80, 5627 (1998).

[13] Q. Si, S. Rabello, K. Ingersent, and J. L. Smith, Nature (London) 413, 804 (2001).

[14] M. J. Besnus, J. P. Kappler, P. Lehmann, and A. Meyer, Solid State Commun. 55, 779 (1985).

[15] H. Kadowaki, M. Sato, and S. Kawarazaki, Phys. Rev. Lett. 92, 097204 (2004), and references therein.

[16] C. Sekine, T. Sakakibara, H. Amitsuka, Y. Miyako, and T. Goto, J. Phys. Soc. Jpn. 61, 4536 (1992).

[17] S. Kawarazaki, M. Sato, H. Kadowaki, Y. Yamamoto, and Y. Miyako, J. Phys. Soc. Jpn. 66, 2473 (1997).

[18] Y. Tabata, T. Taniguchi, M. Sato, S. Kawarazaki, and Y. Miyako, J. Phys. Soc. Jpn. 67, 2484 (1998).

[19] T. Taniguchi, Y. Tabata, and Y. Miyako, J. Phys. Soc. Jpn. 68, 2026 (1999).

[20] Y. Tabata, D. R. Grempel, M. Ocio, T. Taniguchi, and Y. Miyako, Phys. Rev. Lett. 86, 524 (2001).

[21] S. Sachdev, Quantum Phase Transitions (Cambridge University Press, Cambridge, 1999), Chap. 12; Eq. (12.22) in this book corresponds to Eq. (1) (note that $\left.\Gamma\left(\boldsymbol{k}_{3}\right)=D_{c} \kappa_{c}^{2}\right)$.

[22] S. Raymond, L. P. Regnault, J. Flouquet, A. Wildes, and P. Lejay, J. Phys. Condens. Matter 13, 8303 (2001).

[23] We adopt Eqs. 2a and (2b) using a quadratic expansion of Eq. (2.7) in Ref. [6].

[24] H. Kadowaki et al., Phys. Rev. B 68, 140402(R) (2003).

[25] W. Knafo et al., Phys. Rev. B 70, 174401 (2004). 
[26] B. Fåk et al., J. Phys. Condens. Matter 17, 301 (2005).

[27] G. Aeppli et al., Science 278, 1432 (1997).

[28] W. Bao et al., Phys. Rev. B 58, 12727 (1998). 